\title{
Effects of Juglans regia Root Bark Extract on Platelet Aggregation, Bleeding Time, and Plasmatic Coagulation: In Vitro and Ex Vivo Experiments
}

\author{
A. Amirou, ${ }^{1}$ M. Bnouham $\mathbb{D}^{1},{ }^{1}$ A. Legssyer, ${ }^{1}$ A. Ziyyat $\mathbb{D},{ }^{1}$ M. Aziz $\mathbb{D}^{1},{ }^{1}$ \\ M. Berrabah, ${ }^{2}$ and H. Mekhfi ${ }^{1}{ }^{1}$ \\ ${ }^{1}$ Laboratory of Physiology, Genetic and Ethnopharmacology, Faculty of Sciences, Mohammed the First University, Oujda, Morocco \\ ${ }^{2}$ Laboratory of Mineral Solid and Analytical Chemistry, Department of Chemistry, Faculty of Sciences, \\ Mohammed the First University, Oujda, Morocco \\ Correspondence should be addressed to H. Mekhfi; hmekhfi@yahoo.fr
}

Received 22 January 2018; Revised 6 April 2018; Accepted 10 April 2018; Published 14 August 2018

Academic Editor: Vincenzo De Feo

Copyright (c) 2018 A. Amirou et al. This is an open access article distributed under the Creative Commons Attribution License, which permits unrestricted use, distribution, and reproduction in any medium, provided the original work is properly cited.

\begin{abstract}
Platelets have an important role in thrombosis and haemostasis. Hyperactivity of the platelets has been associated with thromboembolic diseases and represents the main cause of complications of cardiovascular diseases. Crude aqueous extract (CAE) of Juglans regia root bark was evaluated for bleeding time, antiaggregant activity by using agonists, thrombin, ADP, collagen, or arachidonic acid (in vitro and ex vivo), and anticoagulant activity by measuring the clotting parameters: activated partial thromboplastin time, prothrombin time, thrombin time, and fibrinogen dosage (in vitro and ex vivo). The result of this study reported that the strongest antiaggregant effect of CAE in vitro was observed on the ADP-induced aggregation with inhibitions up to $90 \%$, while, in ex vivo experiments, the inhibition (more than $80 \%$ ) was observed with all agonists. Anticoagulant effect of CAE significantly prolonged the TT and decreased the fibrinogen level in vitro and ex vivo without interfering with APTT and PT. The bleeding time in mice and rats was significantly increased by CAE. The antiplatelet and anticoagulant effect observed in this study suggest that Juglans regia could have antithrombotic and/or thrombolytic activities and provide an alternative therapy against thrombotic complications related to cardiovascular diseases.
\end{abstract}

\section{Introduction}

Thrombosis is the formation of blood clots (thrombus) in the circulatory system caused by the imbalance of homeostatic system. The majority of cardiovascular diseases, including acute coronary syndrome, venous thromboembolism, deep vein thrombosis, pulmonary embolism, myocardial infarction, atherosclerosis, and ischemic stroke, are associated with thrombotic disorders, resulting in serious consequences such as sudden death $[1,2]$.

The therapies with antithrombotic, anticoagulant, and antiaggregant drugs are still widely used for prevention or for treatment of thrombotic diseases, despite side effects like gastrointestinal symptoms and hemorrhage [3]. Nowadays, scientist carried so much interest toward the development of new antithrombotic agents from the natural products, especially when a lot of people around the world use plants as a remedy against various diseases $[4,5]$. Many plants have been investigated in our laboratory for their antiaggregant potential such as Petroselinum crispum [6] and Argania spinosa [7]. Jin et al. [8] reported that several antithrombotic agents have been explored like Thymus vulgaris, Rosmarinus officinalis, Lavandula hybrida, Allium cepa, Ginkgo biloba, and Olea europaea.

Juglans regia (Jr) or walnut (family of Juglandaceae) is commonly known in Morocco as "Gargae" or "Jawz" for the fruit and "Souak" for the root bark. All parts (bark, leaves, flowers, seed, and fruit) have been widely used in folk medicine to treat many health disorders. Bnouham et al. [9] reported in their reviews that the fruit of $J r$ is used traditionally in Morocco as hypoglycemic, anthelmintic, antiseptic, tonic, and astringent, and the leaves and cortex 
were indicated as depurative, cicatrizing, stomach disorders, and antidote poison. Also, Merzouki et al. [10], in their survey, mentioned that the root bark was prescribed for tooth care and gingivitis.

Experimentally, biological activities of $J r$ have been reported in several reviews, such antioxidant and antitumor, anti-inflammatory, anticarcinogenic, antihyperglycemic activities, and cardioprotective properties [11, 12]. Also, Nergiz-Ünal et al. [13] reported that the dietary intake of walnut reduces the atherosclerotic plaque formation in ApoE-deficient mice. In addition to that, Rywaniak et al. [14] mentioned that extract of $J r$ husks significantly reduced ADPinduced platelet aggregation in whole blood.

However, no study has yet been performed on the antiplatelet and anticoagulant activities of $J r$ root bark. The aim of the present study was to investigate the effect of CAE of Jr root bark on platelet aggregation, coagulation, and bleeding time, in in vitro and in ex vivo experiments.

\section{Materials and Methods}

2.1. Plant Collection. The root bark of walnut (Juglans regia L, Juglandaceae) was collected in January 2014, M'semrir (province of Tinghir in the Southern Morocco). The plant was identified by Professor Mohammed Fennane, an expert botanist from the Scientific National Institute, Rabat (Morocco), and a voucher specimen (HUMPO 149) was deposited in the herbarium at University Mohammed the First (Oujda, Morocco). The bark of Jr was washed with water and dried with air in dark place. The dried bark was powdered by Moulinex Blender and kept in a dark until time of use.

2.2. Crude Aqueous Extract Preparation. The CAE was prepared by infusion of dried bark of $J r(25 \mathrm{~g})$ with boiling distilled water $(500 \mathrm{ml})$ for 30 minutes. The infusate was filtered and evaporated by rotary evaporation (Heidolph Instruments, Germany) at a temperature of $45^{\circ} \mathrm{C}$. The yield of extraction was $11.8 \%$.

2.3. Experimental Animals. Wistar rats and albino mice were housed in an animal unit (Faculty of Sciences, Oujda, Morocco). They were maintained under standard laboratory conditions ( $12 \mathrm{~h}$ light/dark cycle, temperature $22 \pm 2^{\circ} \mathrm{C}$, and with free access of food and water). Experimental protocols were in compliance with the Guide for the Care and Use of Laboratory Animals of the US Department of Health and Human Services (NIH publication no 85-23, revised 1985).

\subsection{In Vitro Antiplatelet Activity}

2.4.1. Washed Platelets Preparation. Washed platelets (WP) were prepared as described by Mekhfi et al. [7]. Animals were slighting anesthetized with ether. Blood was taken by catheterization from the abdominal aorta and deposited into a plastic tube containing anticoagulant solution (9:1, v/v) (citric acid $130 \mathrm{mM}$, trisodium citrate $170 \mathrm{mM}$, and dextrose $4 \%$ ). The blood was immediately centrifuged at $230 \mathrm{~g}$ for 15 min to separate the plasma rich platelet (PRP). PRP was recuperated and centrifuged for $8 \mathrm{~min}$ at $120 \mathrm{~g}$ to eliminate residual blood cells and for $15 \mathrm{~min}$ at $400 \mathrm{~g}$ to obtain the platelet pellet. Then, the platelet pellet was suspended in the wash buffer $\left(\mathrm{NaCl} 137 \mathrm{mM}, \mathrm{KCl} 2.6 \mathrm{mM}, \mathrm{NaHCO}_{3} 12 \mathrm{mM}\right.$, $\mathrm{MgCl}_{2} 0.9 \mathrm{mM}$, Glucose $5.5 \mathrm{mM}, \mathrm{CaCl}_{2} 1.3 \mathrm{mM}$, Gelatin $0.25 \%$, and $\mathrm{pH} 6.5$ ) and centrifuged at $400 \mathrm{~g}$ for $15 \mathrm{~min}$. Finally, the platelets were resuspended in an adequate volume of the final buffer $\left(\mathrm{NaCl} 137 \mathrm{mM}, \mathrm{KCl} 2.6 \mathrm{mM}, \mathrm{MgCl}_{2}\right.$ $0.9 \mathrm{mM}$, Glucose $5.5 \mathrm{mM}, \mathrm{CaCl}_{2} 1.3 \mathrm{mM}$, Gelatin 0.25\%, Hepes $5 \mathrm{mM}$, and $\mathrm{pH}$ 7.4) to make a WP suspension with approximately $5 \times 10^{5}$ cells $/ \mathrm{mm}^{3}$. The prepared WP were used immediately in the same day.

2.4.2. Platelet Aggregation Study. Platelet aggregation studies were performed using an aggregometer (Chrono-Log, Aggregometer, Kordia, USA). Aggregation was measured by the change in light transmission, with the value for the blank sample (buffer without platelets) set at 100\%. For the control test, an aliquot of WP $(250 \mu \mathrm{l})$ was incubated at $37^{\circ} \mathrm{C}$ with $1000 \mathrm{rpm}$. After the incubation period, platelet aggregation was induced by the addition of either final concentration: thrombin $0.5 \mathrm{U} / \mathrm{ml}$, ADP $5 \mu \mathrm{M}$, and collagen $5 \mu \mathrm{g} / \mathrm{ml}$. Aggregation signal was recorded for $5 \mathrm{~min}$ on a paper recorder (Leybold-Heraeus, Austria). For plant tests, WP $(250 \mu \mathrm{l})$ were preincubated with the CAE of $J r(1 \mathrm{mg} / \mathrm{ml})$ for $1 \mathrm{~min}$ at $37^{\circ} \mathrm{C}$ and then activated by agonists.

The parameters measured are as follows:

(i) The amount of aggregation (\%).

(ii) The inhibition of platelet aggregation (Y, \%) calculated using the following equation:

$$
\mathrm{Y}(\%)=[(\mathrm{A}-\mathrm{B}) / \mathrm{A}] \times 100 \text {, }
$$

where $\mathrm{A}$ is the maximum aggregation of the control and $\mathrm{B}$ is the maximum aggregation with plant extract.

2.5. Bleeding Time Assay. Albino mice male and female (18$22 \mathrm{~g}$ ) were randomly divided into four groups of 5 animals per group, treated orally as follows: control group received distilled water $(1 \mathrm{ml} / 100 \mathrm{~g})$, groups of CAE received $J r$ ( 1 and $1.5 \mathrm{~g} / \mathrm{kg}$ ), and the last group received acetyl salicylic acid (ASA) (30 mg/Kg/day). One hour after a single administration, animals were anaesthetised intraperitoneally by sodium pentobarbital $(50 \mathrm{mg} / \mathrm{kg})$ and placed on a hotplate. Bleeding was assessed by amputating $1.5 \mathrm{~cm}$ of the tail tip with a scalpel and blood was blotted into a filter paper. The time between amputation and bleeding cessation was recorded as the bleeding time (BT).

\subsection{In Vitro Anticoagulant Activity}

2.6.1. Preparation of Plasma Sample. This study was realized on plasmatic samples. After anesthesia of the rats (250-300 g) with ether, blood was collected from the abdominal aorta directly into citrated tubes (trisodium citrate $3.8 \%, 1 / 9 ; \mathrm{v} / \mathrm{v}$ ). Blood was immediately centrifuged at $3000 \mathrm{rpm}$ for $20 \mathrm{~min}$, to separate the blood cells from platelet poor plasma (PPP). The PPP was collected and deposited in a plastic tube. 
TABLE 1: Effect of the CAE of Juglans regia on APTT, PT, and TT and fibrinogen concentration.

\begin{tabular}{|c|c|c|c|c|c|}
\hline Sample & Doses & PT (s) & APTT (s) & TT (s) & Fibrinogen $(\mathrm{g} / \mathrm{l})$ \\
\hline Control & $(1: 1 / \mathrm{v}, \mathrm{v})$ & $16.62 \pm 1.16$ & $13.96 \pm 0.57$ & $43.29 \pm 2.20$ & $1.17 \pm 0.09$ \\
\hline \multirow{3}{*}{ Juglans regia $(\mathrm{mg} / \mathrm{ml})$} & 0.25 & $20.12 \pm 1.13^{\mathrm{NS}}$ & $19.74 \pm 1.27^{\mathrm{NS}}$ & $69.5 \pm 1.67^{*}$ & $0.68 \pm 0.05^{* * *}$ \\
\hline & 0.5 & $19.62 \pm 1.53^{\mathrm{NS}}$ & $19.18 \pm 1.55^{\mathrm{NS}}$ & $80.24 \pm 3.21^{* *}$ & $0.64 \pm 0.04^{* * *}$ \\
\hline & 1 & $20.55 \pm 0.76^{*}$ & $25.06 \pm 3.54^{* *}$ & $190.8 \pm 11.63^{* * *}$ & $0.49 \pm 0.02^{* * *}$ \\
\hline Heparin (U/ml) & 0.4 & $53.48 \pm 4.4^{* * *}$ & $>300$ & $>300$ & $0.53 \pm 0.03^{* * *}$ \\
\hline
\end{tabular}

2.6.2. Experimental Protocols. Plasma coagulation experiments were performed by using a semiautomatic coagulometer (Thrombostat, Behnk Elektronik, Norderstedt, Germany).

Plasma mixtures $(100 \mu \mathrm{l}$ of the PPP with $50 \mu \mathrm{l}$ of different concentrations of CAE of $\operatorname{Jr}(0.25,0.5$, and $1 \mathrm{mg} / \mathrm{ml})$ or $50 \mu \mathrm{l}$ of distilled water) were incubated at $37^{\circ} \mathrm{C}$ for 5 min before performing activated partial thromboplastin time (APTT), prothrombin time (PT), and thrombin time (TT) assays. For the fibrinogen assay, PPP was diluted with a suitable buffer. An aliquot of $100 \mu \mathrm{l}$ of this PPP was incubated, for $300 \mathrm{~s}$, with $100 \mu \mathrm{l}$ of CAE of Jr at $37^{\circ} \mathrm{C}$, and then the time of clot formation was measured. Determination of clotting times (APTT, PT, and TT) and fibrinogen level were carried out in accordance with the manufacturer's recommended protocols. Heparin $(0.4 \mathrm{U} / \mathrm{ml})$ was used as positive control for coagulation test.

2.7. Ex Vivo Experiments. Wistar rats (120 - 210 g) were subjected to subchronic treatment. Animals were divided randomly into four groups:

(1) Control group receiving distilled water $(1 \mathrm{ml} / 100 \mathrm{~g}$ /day).

(2) Test group receiving the CAE of $J r(250 \mathrm{mg} / \mathrm{Kg} /$ day $)$.

(3) Positive control group receiving ASA (30 mg/Kg/day) for tail bleeding time and aggregation tests.

(4) Positive control group receiving warfarin $(1 \mathrm{mg} / \mathrm{Kg})$ for coagulation test (a single administration).

All doses were administered orally every morning for 30 days.

2.7.1. Bleeding Time Assay. One hour after the last administration, animals were anesthetized by intraperitoneal injection of sodium pentobarbital $(50 \mathrm{mg} / \mathrm{Kg}$ ) and placed on a hotplate at $37^{\circ} \mathrm{C}$. Bleeding was assessed by amputating $5 \mathrm{~mm}$ of the tail tip and the duration between tail incision and bleeding stops is the bleeding time (s).

After BT determination, the blood was collected into two tubes, used for aggregation and coagulation.

2.7.2. Platelet Aggregation Study. Whole blood was collected, $\mathrm{WP}$ were prepared, and platelet aggregation was performed as described above.

2.7.3. Plasmatic Coagulation Assay. PPP, obtained after centrifugation, was used for the clotting times (APTT, PT, and TT) and fibrinogen determination. These measurements were performed for each group (control and treated), using a commercial diagnostics kits by following the manufacturers' instructions.

Reagents. ADP was purchased from Verum Diagnostica $\mathrm{GmbH}$ (Munich, Germany), collagen calf skin type III from Sigma (USA), thrombin from Sigma (Germany), arachidonic acid from CALBIOCHEM (USA) or Cayman Chemical Company (USA), and warfarin from Sigma (Canada).

Statistical Analysis. Statistical analysis data were performed by using GraphPad Prism Software version 5.01 (GraphPad Software, Inc.). Statistical significance between two groups was determined using Student's t-test. For the treatment studies, the analysis of variance (ANOVA) followed by the Dunnett's test was used. All results are expressed mean \pm SEM and " $p$ " values lower than 0.05 were considered statistically significant.

\section{Result}

3.1. Antiaggregant Effect of Aqueous Juglans regia Bark Extract. After preincubation with the CAE of $J r$ bark for $1 \mathrm{~min}$, platelets were stimulated by $\operatorname{ADP}(5 \mu \mathrm{M})$, thrombin $(0.5$ $\mathrm{U} / \mathrm{ml})$, or collagen $(5 \mu \mathrm{g} / \mathrm{ml})$. Figure 1 showed that the CAE $(1$ $\mathrm{mg} / \mathrm{ml}$ ) inhibits platelet aggregation induced in vitro by ADP, thrombin, or collagen. The percentage of inhibition was 92 $\pm 3 \%, 51.14 \pm 4.19 \%$, and $44.45 \pm 8 \%$, respectively $(n=5)$. Regarding agonists, the strong inhibition of aggregation $(\mathrm{p}<$ $0.001)$ was observed with ADP then thrombin $(\mathrm{p}<0.05)$ and collagen (no significant with $\mathrm{p}>0.05$ ).

3.2. Effect of Juglans regia CAE on Tail Bleeding Time. The BT was measured in vivo, one hour after the oral administration of distilled water, CAE of $J r$, and ASA. As shown in Figure 2, the two doses of $J r$ bark, 1 and $1.5 \mathrm{~g} / \mathrm{Kg}$, significantly ( $\mathrm{p}<$ $0.001)$ prolonged the BT $(317.3 \pm 27.7 \mathrm{~s}$ and $354.5 \pm 23.5 \mathrm{~s}$, respectively), compared to the control group (154.2 \pm 20.8 $\mathrm{s})$. Also, the ASA significantly $(\mathrm{p}<0.001)$ prolonged the BT $(342.1 \pm 23.5 \mathrm{~s})$.

3.3. Anticoagulant Effect of CAE of Juglans regia Bark. Anticoagulant effect of CAE $(0.25,0.5$, and $1 \mathrm{mg} / \mathrm{ml})$ was explored by evaluating coagulation times (PT, APTT, and TT) and fibrinogen concentration in PPP from rats. As shown in Table 1, the CAE of $J r$ at 0.25 and $0.5 \mathrm{mg} / \mathrm{ml}$ prolonged 

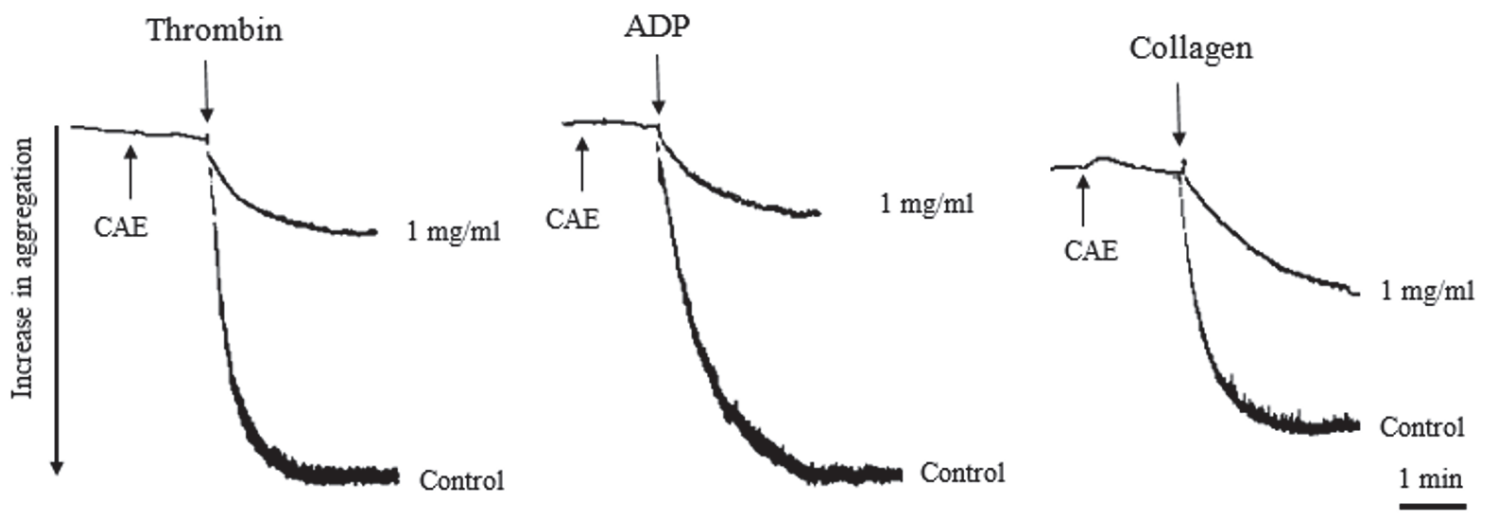

(a)

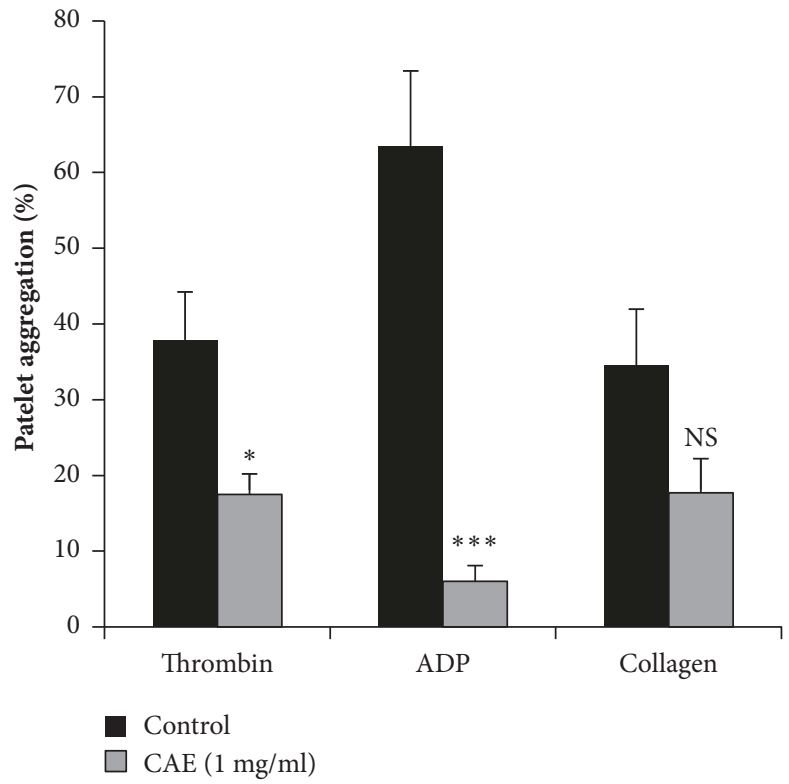

(b)

FIGURE 1: Effect of Juglans regia CAE $(1 \mathrm{mg} / \mathrm{ml})$ on platelet aggregation induced in vitro by thrombin $(0.5 \mathrm{U} / \mathrm{ml})$, ADP $(5 \mu \mathrm{M})$ or collagen $(5 \mu \mathrm{g} / \mathrm{ml}) .{ }^{*} \mathrm{p}<0.05 ;{ }^{* * *} \mathrm{p}<0.001$ compared to control; NS: not significant; $\mathrm{n}=5$. (a) Original tracing and (b) histograms showed the aggregation platelet.

significantly the TT $(\mathrm{p}<0.05$ and $\mathrm{p}<0.01$, respectively) and reduced the fibrinogen concentration $(p<0.001)$ when compared to the control. The PT and APTT still unchanged ( $p>0.05)$, while, at $1 \mathrm{mg} / \mathrm{ml}$ of CAE, all clotting times, PT $(\mathrm{p}<0.05)$, APTT $(\mathrm{p}<0.01)$, and TT $(\mathrm{p}<0.001)$, were extended and the plasmatic fibrinogen level was reduced $(\mathrm{p}<0.001)$. As expected, heparin $(0.4 \mathrm{U} / \mathrm{ml})$, used as anticoagulant drug reference, affects $(\mathrm{p}<0.001)$ in the same manner all these parameters.

\subsection{Ex Vivo Experiments}

3.4.1. Effect of CAE of Jr on Ex Vivo Bleeding Time. The BT of rats was measured to determine the effect of CAE of $J r$ bark $(250 \mathrm{mg} / \mathrm{Kg} /$ day $)$ on platelet function after 4 weeks of treatment. The result shows that BT was significantly prolonged $(\mathrm{n}=5)$ by CAE of $\operatorname{Jr}(304.32 \pm 24.72 \mathrm{~s}, \mathrm{p}<0.01)$ as well as ASA $(327.12 \pm 9.914 \mathrm{~s}, \mathrm{p}<0.001)$ when compared to control (194.4 $\pm 5.87 \mathrm{~s})$.

3.4.2. Antiaggregant Effect of the CAE of Jr Ex Vivo. The CAE of $J r$ effect $(250 \mathrm{mg} / \mathrm{Kg} /$ day $)$ was evaluated on rat platelet aggregation after 4 weeks of treatments. Figure 3 shows that the extract as well as ASA $(30 \mathrm{mg} / \mathrm{Kg} /$ day $)$ reduced significantly $(\mathrm{p}<0.001)$ the amount of platelet aggregation induced by thrombin, ADP, collagen, or arachidonic acid compared to the control group. In all cases, the inhibition of aggregation was more than $85 \%$.

3.4.3. Ex Vivo Anticoagulant Effect of CAE of Jr. The APTT, PT, TT, and fibrinogen concentration were measured in PPP to assess the ex vivo anticoagulant activity of CAE. As shown in Table 2, the bark of Jr significantly prolonged the TT $(\mathrm{p}<0.01)$ and decreased the fibrinogen level $(\mathrm{p}<$ 
TABLE 2: Effect of CAE of $J r$ on ex vivo on PT, APTT, and TT and fibrinogen concentration.

\begin{tabular}{lccccc}
\hline Sample & Doses & PT $(\mathbf{s})$ & APTT $(\mathbf{s})$ & TT (s) & Fibrinogen $(\mathrm{g} / \mathbf{l})$ \\
\hline Distilled water $(\mathbf{m l} / \mathbf{1 0 0 g})$ & 1 & $18.26 \pm 1.51$ & $16.40 \pm 1.46$ & $39.62 \pm 7.39$ & $2.58 \pm 0.07$ \\
\hline CAE of $\boldsymbol{~} \boldsymbol{r}(\mathbf{m g} / \mathbf{K g})$ & 250 & $20.94 \pm 0.39^{\mathrm{NS}}$ & $17.48 \pm 1.81^{\mathrm{NS}}$ & $54.96 \pm 3.46^{* *}$ & $1.98 \pm 0.10^{* * *}$ \\
\hline Warfarin $(\mathbf{m g} / \mathbf{K g})$ & 1 & $33.30 \pm 3.44^{* *}$ & $36.94 \pm 3.06^{* * *}$ & $117.96 \pm 10.27^{* * *}$ & $1.55 \pm 0.06^{* * *}$ \\
\hline
\end{tabular}

Mean \pm SEM, ${ }^{* *} \mathrm{p}<0.01,{ }^{* * *} \mathrm{p}<0.001$, NS: not significant, and $\mathrm{n}=5$.

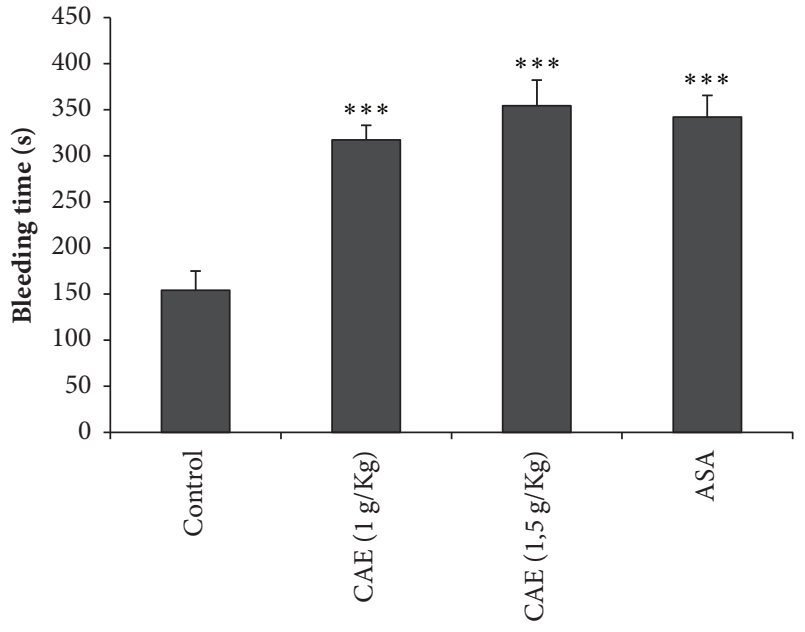

Figure 2: Effect of CAE on tail bleeding time in mice; ${ }^{* * *} \mathrm{p}<0.001$ compared to control; $\mathrm{n}=5$.

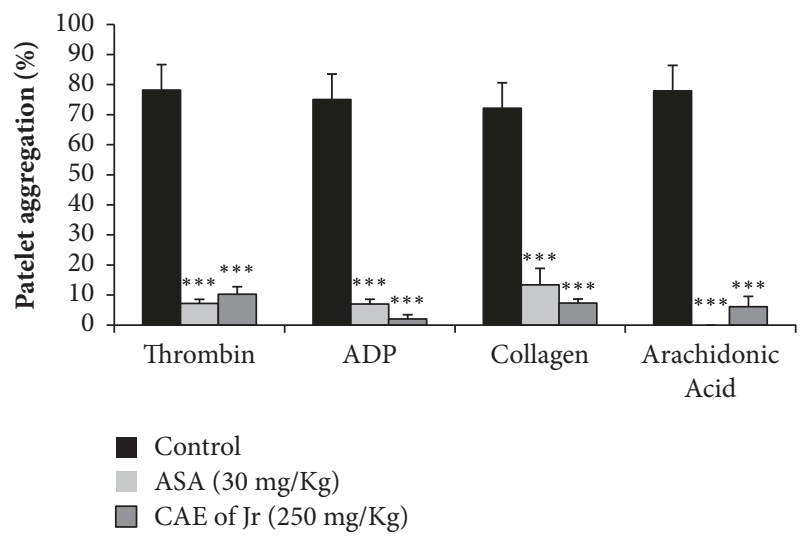

FIGURE 3: Effect of CAE of Jr and ASA on ex vivo platelet aggregation induced by thrombin $(0.5 \mathrm{U} / \mathrm{ml})$, ADP $(5 \mu \mathrm{M})$, collagen $(5 \mu \mathrm{g} / \mathrm{ml})$, and arachidonic acid $(20 \mu \mathrm{M})$. Mean $\pm \mathrm{SEM} ;{ }^{* * *} \mathrm{p}<0.001 ; \mathrm{n}=5$.

0.001), but still without any significant effect on PT and APTT when compared to the control group. Warfarin, used as an anticoagulant drug in the prevention of blood clots formation, significantly $(\mathrm{p}<0.001)$ prolonged all the clotting times and decreased fibrinogen level.

\section{Discussion}

Platelets play a critical role in hemostasis and their major purpose is to plug the injuries in the vessel walls. They are also an important contributor in the development of abnormal thrombosis that is related to the cardiovascular diseases [15]. Despite the existence of a well know antithrombotic remedies with a proven efficacy, the research of novel bioactive natural products interfering with platelets and/or plasmatic coagulation is increasingly intense. The optimal bioactive compound may be efficient but with less side effects like bleeding.

In this study, we presented crude aqueous extract of Juglans regia root bark as a potent extract that affects primary and secondary haemostasis. The primary haemostasis is commonly explored by platelet aggregation and bleeding time measurements, while the secondary haemostasis is investigated by plasmatic coagulation times (PT, APTT, and TT) and fibrinogen determinations. In the in vitro experiments, the obtained data show that CAE of $J r$ at $1 \mathrm{mg} / \mathrm{ml}$ exhibits a strong inhibition of platelet aggregation induced by ADP, thrombin, not by collagen, and a significant prolongation of $\mathrm{BT}$. In practice, BT is a useful test to detect abnormal platelet function. Its extension in our study confirms the antiaggregant activity of $J r$ extract. Scientific literature reported that several plants have shown inhibition of platelet aggregation with or without affecting BT such as Kyung-Ok-Ko (mixture of several medicinal plants) [16], Lagenaria siceraria [17], argan oil [7], Asarum sieboldii [18], Geoffroea spinosa [19], and Mucuna pruriens [20]. In general, speculations about molecular mechanism explaining the antiaggregant activity evoke many signaling pathways, including those mediated by phospholipase C (PLC) [21], phospholipase A2 (PLA2) and thromboxane A2 (TXA2), or the stimulation of adenylate cyclase (cAMP) and guanylate cyclase (cGMP) [22]. In ex vivo results, the antiaggregant effect of $J r$ extract was observed with all used platelets activators: ADP, thrombin, collagen, and arachidonic acid. It is well known that each agonist attaches to its specific platelet receptor, then mobilizes intracellular messengers (cAMP, cGMP), and activates enzymes. Bioactive compounds, present in $J r$ extract, may probably interfere negatively with ADP (P2Y1, P2Y12), thrombin (PAR 1 and PAR 2), and collagen or arachidonic receptors. Theses interactions cause finally a fall in intracellular calcium. In the coagulation cascade (intrinsic, extrinsic, and common phases), parameters such as PT, APTT, TT, and fibrinogen concentration are the basic blood tests for evaluating hemorrhage and thrombosis risk. APTT is related to the intrinsic and/or common pathways of plasmatic coagulation and is used to detect deficiencies of factors II, V, VIII, IX, X, XI, and XII. PT explores the extrinsic phase for detecting bleeding disorders and affected clothing factors in the extrinsic or common pathways. TT and fibrinogen amount concern the common and ultimate coagulation phase and their determinations explore the ability of thrombin to catalyze the polymerization of fibrinogen to form fibrin 
$[23,24]$. Our experiments demonstrate that CAE of $J r$, at lower concentrations, significantly extends TT and diminishes fibrinogen content. But, at $1 \mathrm{mg} / \mathrm{ml}$, all coagulation parameters are modified in the same way. These observations let us to suggest that CAE of plant exerts a strong action on the common pathway, more than the extrinsic and intrinsic pathways of plasmatic coagulation. In this context, the use of heparin as an anticoagulant and antithrombotic drug in therapeutic [25] confirms and validates the $J r$ CAE results. In order to verify if CAE of $J r$ is still efficient in vivo, animals were fed with plant extract for one month and, at the end of treatment, all parameters were performed in ex vivo. The obtained results showed that, in treated rats, CAE exerts significantly the same effects comparing to in vitro studies, suppression of platelet aggregation, prolongations of $\mathrm{BT}$ and TT, and reduction of plasmatic fibrinogen level, while APTT and TP remain unchanged in ex vivo conditions.

The main objective to use the CAE in our investigation is to confirm the medicinal properties of this plant, as possible as, in the same conditions of population use. With this type of extract, it is not easy to relate exactly the observed effects to one or more compounds because the CAE constitute a complex and important mixture of bioactive substances that may probably act in synergy.

Phytochemical analysis of methanolic and aqueous extracts of $J r$ bark revealed the presence of many and diverse compound families [26, 27], among them, polyphenols, which constitute a large group of natural products widely found in different vegetables. This class is divided into several subclasses such as phenolic acids, flavonoids, lignans, and tannins. These compounds are well known by their broad range of cardiovascular activities like vasodilating, antiplatelet aggregation [28]. In their review, Middleton et al. [29] reported several studies related the beneficial effect of flavonoids in hemostasis and thrombosis. These authors mentioned that flavonoids significantly inhibited platelet function (adhesion, aggregation, and secretion). In this domain, the mechanism of action of flavonoids is well documented. Some flavonoid molecules like flavone, chrysin, apigenin, and phloretin act by depressing platelet cyclooxygenase (CO) activity and decreased the cAMP response to prostacyclin [29]. Recently, Du et al. [30] reported that quercetin and rutin are the most flavonoids commonly studied for their cardiovascular effects. Quercetin was an effective inhibitor of lipoxygenase (12-LO) activity in human platelets and its action has been related to the inhibition of arachidonic acid metabolism by CO [29]. Choi et al. [31] demonstrated that rutin inhibited collagen induced platelet aggregation in human platelets and prolonged APTT and PT. Furthermore, Correia-Da-Silva et al. [32] reported that transresveratrol 3$B$-D-glucopyranoside persulfate and sulfated oligoflavonoids have been described as anticoagulant/antiplatelet agents.

The involvement of such compounds in hemostasis was already initiated in our laboratory. Flavonoids (genins and heterosidic flavonoids) isolated from Arbutus unedo [33] or Petroselinum crispum [6] inhibit platelet aggregation. More, Gadi et al. [6] reported that the adhesion of human platelets to collagen under flow was greatly decreased by genins of Petroselinum crispum.

\section{Conclusion}

In the present study, the crude aqueous extract of Juglans regia root bark exerts in vitro and ex vivo potent antiplatelet and anticoagulant effects. These data suggest that Juglans regia could be a promising therapy preventing thrombotic complications of cardiovascular disease.

\section{Data Availability}

All data used to support this study are available to the reader in the Laboratory of Physiology, Genetics and Ethnopharmacology, Faculty of Sciences, Université Mohammed Premier, Oujda, Morocco.

\section{Conflicts of Interest}

The authors declare that they have no conflicts of interest.

\section{Acknowledgments}

This work was supported by grants from the Ministère de l'Éducation Nationale, de l'Enseignement Supérieur, de la Recherche Scientifique et de la Formation des Cadres (Morocco) and the Centre National de la Recherche Scientifique et Technique (Morocco): Project PPR2. The authors thank Professor Mostafa El Achouri (responsible for the herbarium of the University Mohammed the First in Oujda) for his help and thank Mr. Mostafa Bedraoui (Faculty of Sciences, University Mohammed the First in Oujda) for the reliable care of animals breeding.

\section{References}

[1] Y.-N. Shi, Y.-M. Shi, L. Yang et al., "Lignans and aromatic glycosides from Piper wallichii and their antithrombotic activities," Journal of Ethnopharmacology, vol. 162, pp. 87-96, 2015.

[2] Y. Román, H. P. de Oliveira Barddal, M. Iacomini, G. L. Sassaki, and T. R. Cipriani, "Anticoagulant and antithrombotic effects of chemically sulfated fucogalactan and citrus pectin," Carbohydrate Polymers, vol. 174, pp. 731-739, 2017.

[3] M. Struijk, D. F. Postma, S. A. C. Van Tuyl, and M. A. Van De Ree, "Optimal drug therapy after aspirin-induced upper gastrointestinal bleeding," European Journal of Internal Medicine, vol. 23, no. 3, pp. 227-230, 2012.

[4] J.-H. Choi, D.-W. Kim, S.-E. Park et al., "Novel thrombolytic protease from edible and medicinal plant Aster yomena (Kitam.) Honda with anticoagulant activity: Purification and partial characterization," Journal of Bioscience and Bioengineering, vol. 118, no. 4, pp. 372-377, 2014.

[5] S. Mahmud, S. Akhter, M. A. Rahman et al., " Antithrombotic Effects of Five Organic Extracts of Bangladeshi Plants ," Evidence-Based Complementary and Alternative Medicine, vol. 2015, pp. 1-8, 2015.

[6] D. Gadi, M. Bnouham, M. Aziz et al., "Flavonoids purified from parsley inhibit human blood platelet aggregation and adhesion to collagen under flow.", Journal of complementary \& integrative medicine, vol. 9, p. 19, 2012.

[7] H. Mekhfi, D. Gadi, M. Bnouham, A. Ziyyat, A. Legssyer, and M. Aziz, "Effect of argan oil on platelet aggregation and 
bleeding time: A beneficial nutritional property," Journal of Complementary and Integrative Medicine, vol. 5, no. 1, 2008.

[8] W. Y. Jin, S. Kim, H. K. Kim et al., "Antiplatelet and antithrombotic effect of Phyllostachys pubescens leaves and Mume Fructus combination," Integrative Medicine Research, vol. 2, no. 2, pp. 70-75, 2013.

[9] M. Bnouham, H. Mekhfi, A. Legssyer, and A. Ziyyat, "Medicinal plants used in the treatment of diabetes in Morocco," International Journal of Diabetes and Metabolism, vol. 10, no. 1, pp. 3350, 2002.

[10] A. Merzouki, F. Ed-derfoufi, and J. Molero Mesa, "Contribution to the knowledge of Rifian traditional medicine. II: Folk medicine in Ksar Lakbir district (NW Morocco)," Fitoterapia, vol. 71, no. 3, pp. 278-307, 2000.

[11] Nael Abu Taha and M. A. Al-wadaan, "Utility and importance of walnut, Juglans regia Linn: A review," African Journal of Microbiology Research, vol. 5, no. 32, 2011.

[12] N. Panth, K. R. Paudel, and R. Karki, "Phytochemical profile and biological activity of Juglans regia," Journal of Integrative Medicine, vol. 14, no. 5, pp. 359-373, 2016.

[13] R. Nergiz-Ünal, M. J. E. Kuijpers, S. M. De Witt et al., "Atheroprotective effect of dietary walnut intake in ApoEdeficient mice: Involvement of lipids and coagulation factors," Thrombosis Research, vol. 131, no. 5, pp. 411-417, 2013.

[14] J. Rywaniak, B. Luzak, A. Podsedek, D. Dudzinska, M. Rozalski, and C. Watala, "Comparison of cytotoxic and anti-platelet activities of polyphenolic extracts from Arnica montana flowers and Juglans regia husks," Platelets, vol. 26, no. 2, pp. 168-176, 2014.

[15] F. A. Balabin and A. N. Sveshnikova, "Computational biology analysis of platelet signaling reveals roles of feedbacks through phospholipase C and inositol 1, 4, 5-trisphosphate 3-kinase in controlling amplitude and duration of calcium oscillations," Math. Biosci, vol. 276, no. 2016, pp. 67-74, 2016.

[16] T.-H. Kim, K. M. Lee, N. D. Hong, and Y.-S. Jung, "Anti-platelet and anti-thrombotic effect of a traditional herbal medicine Kyung-Ok-Ko," Journal of Ethnopharmacology, vol. 178, pp. 172179, 2016.

[17] M. S. Rajput, N. Balekar, and D. K. Jain, "Inhibition of ADPinduced platelet aggregation and involvement of non-cellular blood chemical mediators are responsible for the antithrombotic potential of the fruits of Lagenaria siceraria," Chinese Journal of Natural Medicines, vol. 12, no. 8, pp. 599-606, 2014.

[18] S.-K. Ku, I.-C. Lee, J. A. Kim, and J.-S. Bae, "Antithrombotic activities of pellitorine in vitro and in vivo," Fitoterapia, vol. 91, pp. 1-8, 2013.

[19] A. C. Sabbione, A. Scilingo, and M. C. Añón, "Potential antithrombotic activity detected in amaranth proteins and its hydrolysates," LWT- Food Science and Technology, vol. 60, no. 1, pp. 171-177, 2015.

[20] F. Herrera Chalé, J. C. Ruiz Ruiz, D. Betancur Ancona, J. J. Acevedo Fernández, and M. R. Segura Campos, "The hypolipidemic effect and antithrombotic activity of Mucuna pruriens protein hydrolysates," Food \& Function, vol. 7, no. 1, pp. 434$444,2016$.

[21] M. Fuentes, C. Sepúlveda, M. Alarcón, I. Palomo, and E. Fuentes, "Buddleja globosa (matico) prevents collagen-induced platelet activation by decreasing phospholipase C-gamma 2 and protein kinase C phosphorylation signaling," Journal of Traditional and Complementary Medicine, 2018.
[22] M. Nardini, F. Natella, and C. Scaccini, "Role of dietary polyphenols in platelet aggregation. A review of the supplementation studies," Platelets, vol. 18, no. 3, pp. 224-243, 2007.

[23] N. Sayari, R. Balti, M. Ben Mansour et al., "Anticoagulant properties and cytotoxic effect against HCT116 human colon cell line of sulfated glycosaminoglycans isolated from the Norway lobster (Nephrops norvegicus) shell," Biomedicine \& Pharmacotherapy, vol. 80, pp. 322-330, 2016.

[24] D.-W. Kim, K. Sapkota, J.-H. Choi, Y.-S. Kim, S. Kim, and S.J. Kim, "Direct acting anti-thrombotic serine protease from brown seaweed Costaria costata," Process Biochemistry, vol. 48, no. 2, pp. 340-350, 2013.

[25] C. Zhu, P. Xu, Y. He et al., "Heparin Increases Food Intake through AgRP Neurons," Cell Reports, vol. 20, no. 10, pp. 24552467, 2017.

[26] V. Upadhyay, S. Kambhoja, and K. Harshaleena, "Antifungal Activity and Preliminary Phytochemical Analysis of Stem Bark Extracts of Juglans regia linn," International Journal Journal of Pharmaceutical and Biological Archive, vol. 1, no. 5, pp. 442-447, 2010.

[27] R. R. Deshpande, A. A. Kale, A. D. Ruikar et al., "Antimicrobial activity of different extracts of Juglans regia L. against oral microflora," International Journal of Pharmacy and Pharmaceutical Sciences, vol. 3, no. 2, pp. 200-201, 2011.

[28] C. Sandoval-Acuña, J. Ferreira, and H. Speisky, "Polyphenols and mitochondria: an update on their increasingly emerging ROS-scavenging independent actions," Archives of Biochemistry and Biophysics, vol. 559, pp. 75-90, 2014.

[29] E. Middleton Jr., C. Kandaswami, and T. C. Theoharides, “The effects of plant flavonoids on mammalian cells: implications for inflammation, heart disease, and cancer," Pharmacological Reviews, vol. 52, no. 4, pp. 673-751, 2000.

[30] G. Du, L. Sun, R. Zhao et al., "Polyphenols: Potential source of drugs for the treatment of ischaemic heart disease," Pharmacology \& Therapeutics, vol. 162, pp. 23-34, 2016.

[31] J.-H. Choi, D.-W. Kim, S.-E. Park et al., "Anti-thrombotic effect of rutin isolated from Dendropanax morbifera Leveille," Journal of Bioscience and Bioengineering, vol. 120, no. 2, pp. 181-186, 2015.

[32] M. Correia-Da-Silva, E. Sousa, B. Duarte, F. Marques, L. M. Cunha-Ribeiro, and M. M. M. Pinto, "Dual anticoagulant/antiplatelet persulfated small molecules," European Journal of Medicinal Chemistry, vol. 46, no. 6, pp. 2347-2358, 2011.

[33] M. El Haouari and H. Mekhfi, "Anti-platelet aggregation effects of extracts from Arbutus unedo leaves," Plant Science Today, vol. 4, no. 2, p. 68, 2017. 


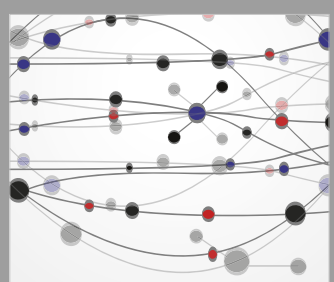

The Scientific World Journal
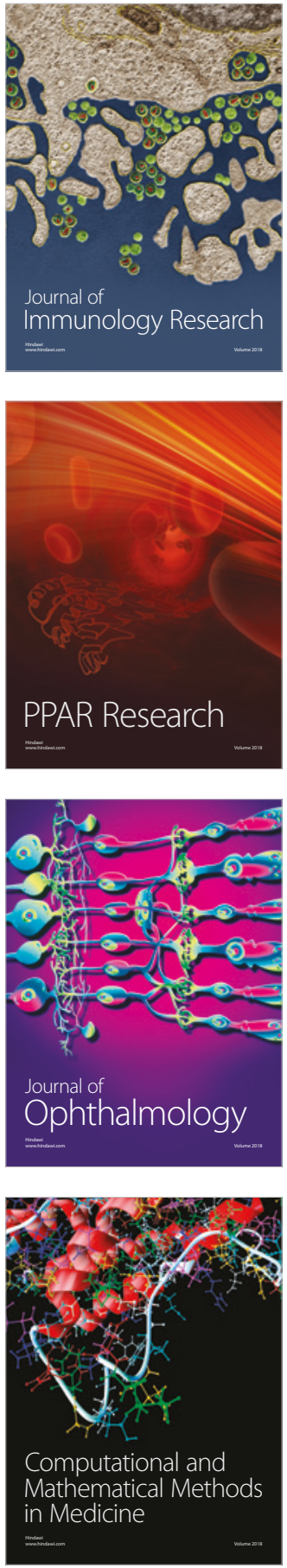

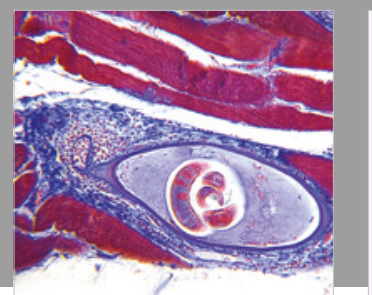

Gastroenterology Research and Practice

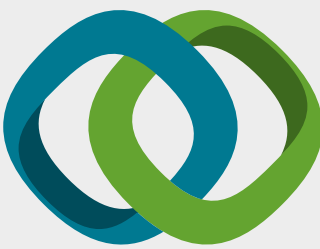

\section{Hindawi}

Submit your manuscripts at

www.hindawi.com
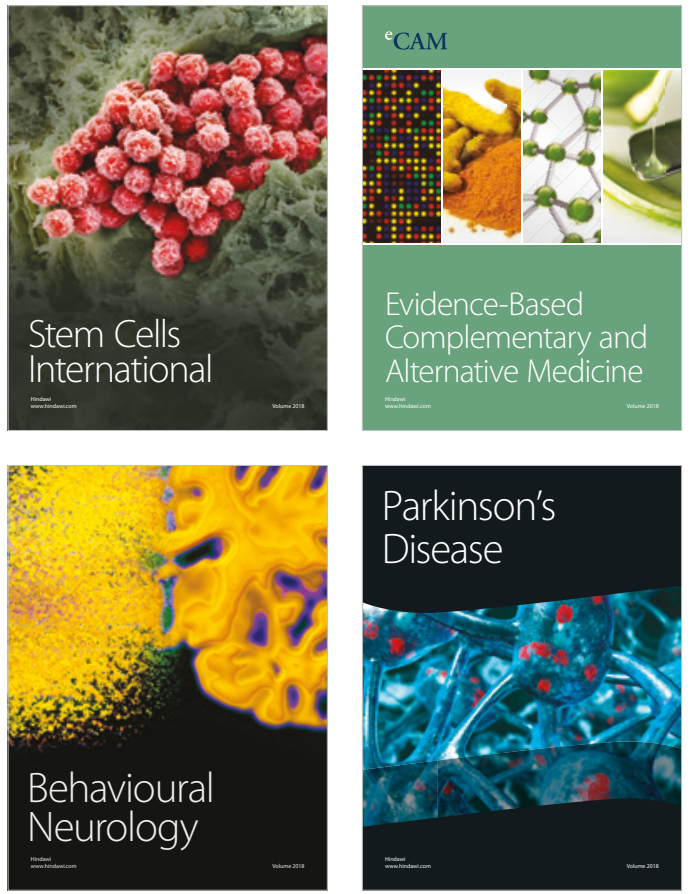

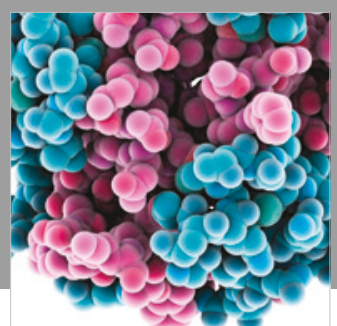

ournal of

Diabetes Research

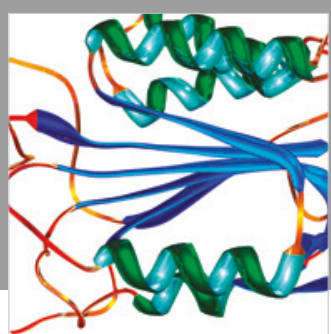

Disease Markers
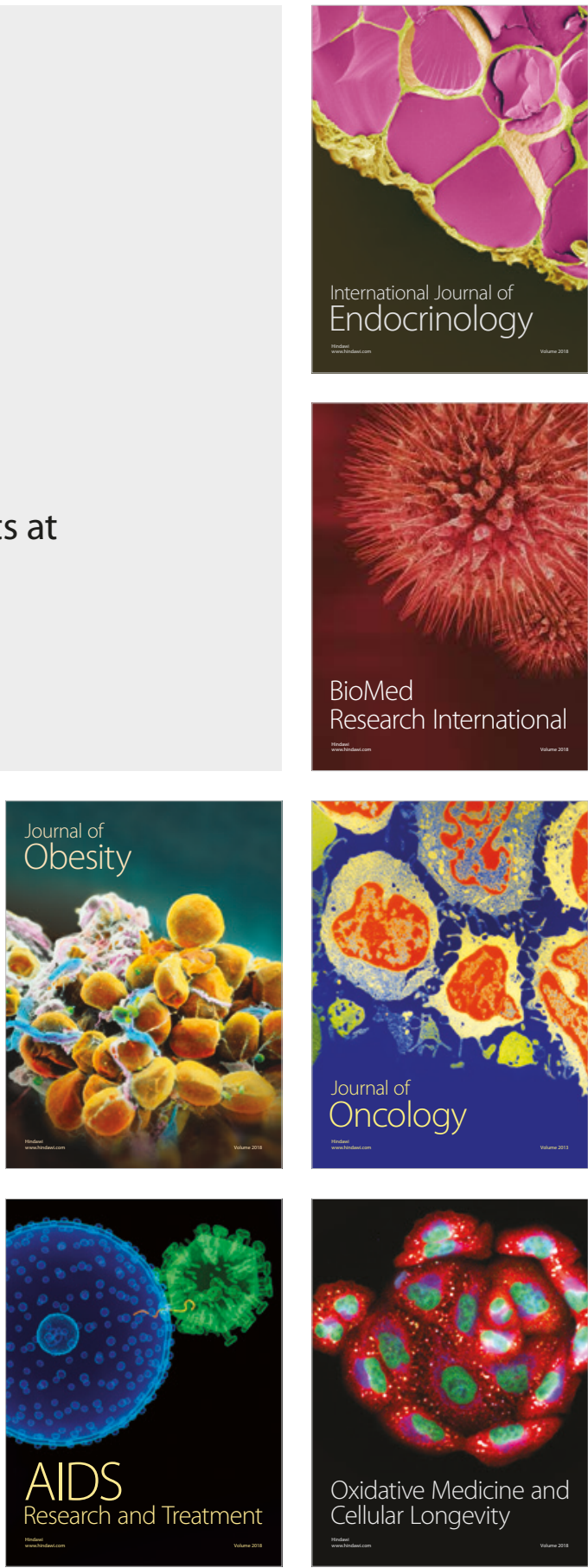